\title{
Pharmacist intervention in the management of Parkinson's disease: evaluating the pharmacist's intervention at a movement disorders outpatient clinic
}

\author{
Akram Shueb
}

Correspondence to

Akram Shueb, Pharmacy

Department, Medicine and

Surgery, University of Malta,

Msida MSD 2080, Malta;

akramshueb@yahoo.com

Received 1 December 2011 Accepted 26 April 2012

\begin{abstract}
Objectives To develop and implement a

pharmacist intervention plan in the management of patients with Parkinson's disease in an outpatient setting.

Methods Thirty-five patients (19 men, 16
\end{abstract} women) with Parkinson's disease were recruited from the Movement Disorders Outpatient Clinic at Zammit Clapp Hospital. An intervention plan was carried out during the first visit followed by evaluation during the second visit after 8 weeks. Intervention tools included a treatment medication chart, two patient leaflets and a pharmacist-run discussion with patients and their caregivers. Outcomes were measured using a Compliance Questionnaire, the PDO-39 Quality of Life Questionnaire and an Intervention Evaluation Sheet. Data collected were entered into a spreadsheet for analysis of descriptive statistics and correlation studies.

Results All participants were Maltese with a mean age of 74 years. During the second visit, a statistically significant improvement was seen in patients' compliance with treatment compared with the first visit $(p=0.000)$. The average domain dimension scores of the PDO-39 questionnaire showed an overall improvement in the patients' quality of life with Mobility $(p=0.038)$, Activities of Daily Living $(p=0.006)$ and Social Support $(p=0.01)$ showing a statistically significant improvement following the pharmacist's intervention. Fifteen patients were in favour of always having a pharmacist intervening.

Conclusions The inclusion of the hospital pharmacist in the multidisciplinary team of healthcare professionals significantly improves patient and caregiver well-being through better medication compliance and improved health status.

\section{INTRODUCTION}

The interplay of patient symptoms, caregiver burden and medication non-compliance all contribute to demanding tasks in Parkinson's disease. Pharmacist intervention is needed to affect patient treatment. The objective of this study was to develop and implement a pharmacist intervention plan for the management of patients with Parkinson's disease in the outpatient setting at Zammit Clapp Hospital, a rehabilitation hospital. The aims of the study were to improve medication compliance, to ameliorate the patients' quality of life (OOL) status, to assess the QOL domains and the relationship between compliance and $\mathrm{QOL}$, and to evaluate the role of pharmacist intervention and the resulting outcome on patient care.

\section{METHODS}

Thirty-five patients with Parkinson's disease were recruited from the outpatient Movement Disorders Clinic at Zammit Clapp Hospital. The patients were men and women of all ages with Parkinson's disease of all stages. The patients participating in the study were either English or Maltese speakers, capable of communicating, and did not have dementia or cognitive impairment.

The intervention tools used included a Treatment Medication Chart (figure 1), two patient leaflets which

\begin{tabular}{|c|c|c|c|c|c|c|}
\hline \multirow[t]{2}{*}{ Regular medication taken } & \multirow[t]{2}{*}{ Strength } & \multirow[t]{2}{*}{ Reason } & \multicolumn{4}{|c|}{ When to take } \\
\hline & & & $\begin{array}{c}\text { os } \\
\text { wAKING }\end{array}$ & $\begin{array}{l}\mathrm{LCSCH} \\
\text { 2PM }\end{array}$ & $\begin{array}{l}\text { IEA } \\
\text { GPM }\end{array}$ & BEDIMIE \\
\hline & & & & & & \\
\hline & & & & & & \\
\hline & & & & & & \\
\hline & & & & & & \\
\hline & & & & & & \\
\hline & & & & & & \\
\hline & & & & & & \\
\hline & & & & & & \\
\hline & & & & & & \\
\hline & & & & & & \\
\hline
\end{tabular}

Figure 1 Treatment Medication Chart. 
were specifically developed for this study (Improving Quality of Life and Tips for Caregivers of Parkinson's Disease Patients, available in both English and Maltese) and a pharmacist-run discussion with patients and their caregivers. The Improving Quality of Life leaflet was composed of a list of advice aimed at improving the patient's OOL. These tips were subdivided into three categories: advice on nutrition, medication and lifestyle. The Tips for Caregivers of Parkinson's Disease Patients leaflet was targeted towards the patients' caregivers. It was composed of a list of caregiver tips to help them cope with the condition of their loved ones, ameliorate their QOL, prevent them from being stressed and educating them on the freedom of need for help and advice at any stage.

Outcomes were measured using three tools taken from previously available instruments: a Compliance Questionnaire, ${ }^{1}$ the PDO-39 Quality of Life Questionnaire ${ }^{2}$ and an Intervention Evaluation Sheet. ${ }^{3}$ The intervention plan (figure 2), which involved the

\section{Phase 1 : INTERVENTION}

- Review of the patient

- Completion of the 'Treatment Medication Chart'

- Completion of the 'Compliance Questionnaire'

- Completion of the 'PDQ-39' Quality of Life Questionnaire

- Administration of the leaflets:

○ 'Improving Quality of Life'

- 'Tips for Caregivers of Parkinson's disease patients'

- Pharmacist-run discussion with patients and their caregivers

Phase 2 : EVALUATION

- Completion of the 'Compliance Questionnaire'

- Completion of the 'PDQ-39' Quality of Life Questionnaire

- Completion of the 'Intervention Evaluation Sheet'

- Pharmacist-run discussion with patients and their caregivers

\section{DISCUSSION}

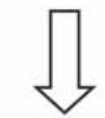

- Results before and after the pharmacist's intervention were analyzed.

- Correlations were made between the patients' demographic data, compliance to medications and their quality of life, together with the pharmacist's intervention criteria.

- The intervention tools served to hypothesise whether the intervention of the pharmacist should be implemented in this setting with the objective to aid in improving the patients' quality of life, coping with medications and consequently offering an additional benefit in the management of Parkinson's disease at the 'Clinic of Movement Disorders'. 
completion of both the compliance and OOL questionnaires and a pharmacist-run interview with the patients, was carried out during the first visit followed by evaluation during the second visit after at least 8 weeks.

Compliance with medications describes the degree to which patients correctly adhere to their treatment and medical advice. OOL is an evaluation of the general well-being of the patient. These two factors were assessed by means of their respective questionnaires and the relationship between the two was later analysed.

Patient demographic data and patient history and drug treatment information were obtained from patient/caregiver interview during the pharmacist-run discussions. Other information (Hoehn and Yahr Disability Scale and Mental Test Score) was collected from the patients' case notes. The Hoehn and Yahr Disability Scale is a Parkinson's disease scale that allocates stages from 0 to 5 in order of increasing disability to indicate the level of disability of the patient, with stage 0 indicating no sign of disease and stage 5 indicating greatest disability. The Mental Test Score is a simple test for quickly assessing the patient's level of confusion or any other cognitive impairment. It comprised 10 simple short questions to the patient such as telling the time, year, age, counting backwards from 20 down to 1, etc. A point was allocated for each correct answer given by the patient and no points were given for wrong or unanswered questions. The total score out of 10 was recorded on the patient's file, lower scores indicating a greater tendency to cognitive impairment.

The data collected were entered in a spreadsheet using SPSS Statistics Version 17.0 and descriptive statistics and correlation studies were analysed. The statistical tests used for the study were the paired samples t test, Pearson correlation test, $\chi^{2}$ test and one-way ANOVA test.

The paired samples t test was used to compare the mean scores of each domain before and after the pharmacist's intervention. The null hypothesis specified that the intervention was not effective, meaning that the average scores before and after intervention were comparable for a particular domain. The alternative hypothesis specified that the intervention was effective.

The Pearson correlation test was used to measure the relationship between two quantitative variables (ie, covariants). A correlation close to 1 indicated a strong positive correlation whereas a correlation close to -1 indicated a strong negative relationship. A correlation close to 0 indicated that there was no relationship. The null hypothesis for the Pearson correlation test specified that there was no relationship between the two variables (ie, correlation close to 0 ). However, the alternative hypothesis specified that there was a significant relationship which was not attributed to chance (ie, correlation significantly different from 0 ).

The $\chi^{2}$ test was used to determine whether there was a statistically significant difference between the expected and observed frequencies in one or more categories. It was used when both results were qualitative and was thus used to check for associations between categorical variables. The null hypothesis for the $\chi^{2}$ test stated that there was no association between the categorical variables so there was no bias between subjects in different groups. However, the alternative hypothesis stated that there was a significant association between the two categorical variables, indicating a significant bias between subjects in different groups.

The one-way ANOVA test was used to compare quantitative with qualitative data, therefore comparing the mean rating score for an item or statement between several independent groups. For the oneway ANOVA test, the null hypothesis stated that the mean rating scores obtained by the independent groups were equal. However, the alternative hypothesis stated that the mean rating scores obtained by some groups were higher than other groups.
The $\mathrm{p}$ value was the criterion used to determine whether to accept the null hypothesis or the alternative hypothesis. If the $\mathrm{p}$ value was $>0.05$ level of significance, the null hypothesis was accepted. Conversely, if the $p$ value was $<0.05$, then the alternative hypothesis was accepted. This applied for all the statistical tests used in the study.

\section{RESULTS}

All 35 recruited patients were Maltese; 19 (54\%) were men and 16 (46\%) were women. The mean age was 74 years (range 65-88) and the mean duration of Parkinson's disease was 6 years (range 5 months to 25 years). With advancing age, the prevalence of being diagnosed with Parkinson's disease and the patients' Hoehn and Yahr scale increased.

The study confirmed the occurrence of mental decline with increasing age, since the Mental Test Score was lower in older patients and the progressive mobility impairment in patients with Parkinson's disease was accompanied by an increased risk of cognitive decline. Patients' level of education varied from primary to tertiary level, but most patients $(n=28,80 \%)$ had received education up to a primary level of education.

All patients enrolled in the study had comorbidities in addition to the main presenting complaint of Parkinson's disease (figure 3). The medications taken consisted of anti-parkinsonian medications together with medications to treat other accompanying conditions; the majority of patients $(n=28,80 \%)$ took $6-10$ medications.

A statistically significant improvement $(p=0.000)$ was evident in patient's adherence to treatment following the pharmacist's intervention (figure 4). Prior to the pharmacist's intervention, 28 patients (80\%) took their medications at their prescribed times. At the second visit all patients claimed to have taken their medications at the correct times. Age was the only factor to yield a significant correlation following the pharmacist's intervention, with younger patients showing better medication compliance than older patients.

The PDO-39 scores ranged from 0 to 100 with a lower score indicating a better OOL. Seven of the eight domains improved during the second visit, with Mobility $(p=0.038)$, Activities of Daily Living (ADL) $(p=0.006)$ and Social Support $(p=0.01)$ showing a statistically significant improvement following the pharmacist's intervention (figure 5).

The PDO-39 scores showed that the patient's OOL was overall better in men than in women, with women having a better $\mathrm{QOL}$ in only three domains (ADL, Cognition and Communication) while men had a better OOL in the Mobility, Emotional, Stigma, Social Support and Bodily Discomfort domains. This was most evident

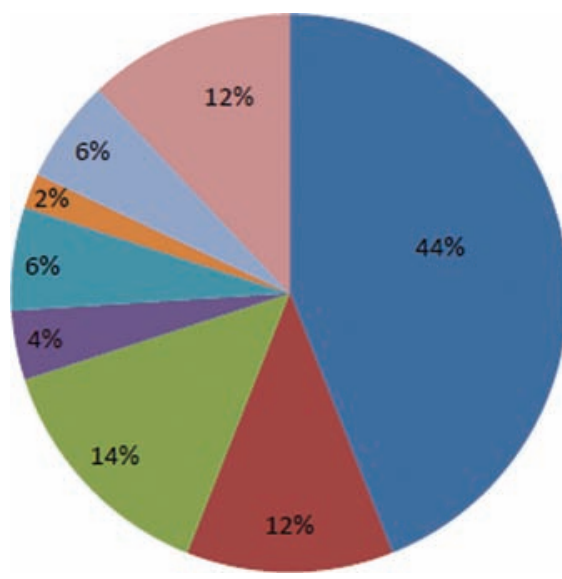

- Heart failure and Hypertension - Depression

- Diabetes and Hyperthyroidism E Schizophrenia Dementia Epilepsy Elaucoma

Prostatic Hyperplasia

Figure 3 Comorbidities of study patients. 


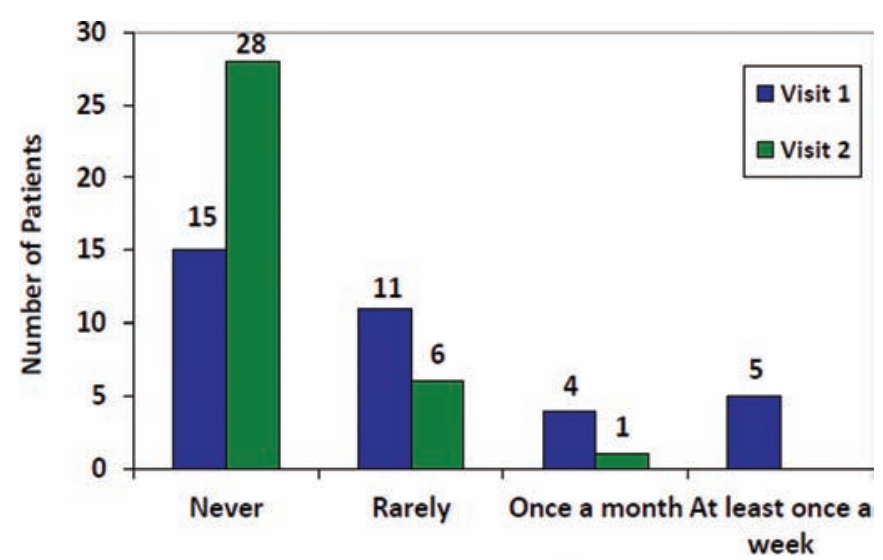

Frequency of Missed Medications

Figure 4 Patients' adherence to treatment at visits 1 and 2.

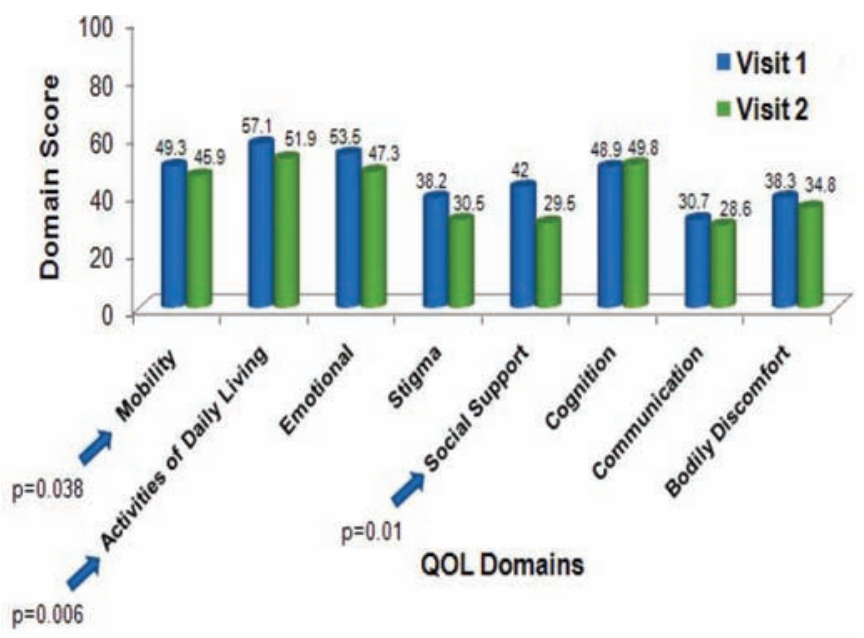

Figure 5 PDQ-39 quality of life (OOL) scores at visits 1 and 2.

for the domain Emotional where women were found to experience significantly $(p=0.046)$ poorer emotional well-being than men, indicating that $\mathrm{QOL}-$ and particularly emotional status-can vary by gender. This was supported by previous studies which showed that women were more likely to experience complications from Parkinson's disease including impact on psychiatric aspects and unwanted effects of drug treatment. ${ }^{4}$

When the OOL domains were compared with age it was found that the majority of domains showed a positive correlation with advancing age. This signified that older patients generally had a lower QOL as they scored higher in the PDO-39. A statistically significant association for this correlation was evident for the domain $\mathrm{ADL}(\mathrm{p}=0.037)$, where the patients' activities of daily living became more impaired with increasing age. Researchers have found that daily activities were the basic ADLs that were first affected, while eating and going to the toilet were the last activities to be affected. Consequently, a patient history regarding his or her abilities to perform daily activities related to personal hygiene, mobility and selfcare can aid the clinician to identify the first signs of Parkinson's disease. $^{5}$

The PDQ-39 domain scores did not significantly demonstrate a superior $\mathrm{OOL}$ in patients with a higher level of education. Results from earlier studies are conflicting in that some studies identified education as relevant for health-related $\mathrm{OOL}^{67}$ while other studies did not show a strong association between the two variables. ${ }^{89}$ Findings from previous studies showed that the $\mathrm{OOL}$ of patients with Parkinson's disease was significantly better when disease duration was shorter. ${ }^{10}$ However, the results from this study did not demonstrate statistically significant differences between the $\mathrm{OOL}$ dimensions and disease duration.

The results comparing the relationship between the PDO-39 QOL domains and the number of medications taken by patients with Parkinson's disease showed that Cognition ( $p=0.038$ before and $p=0.032$ after) and Communication $(p=0.037)$ had a statistically significant positive correlation. This indicates that, as patients take more medications, a higher mean $\mathrm{PDO}$ value is obtained corresponding to a lower QOL. Polypharmacy may be a contributing factor where the result of adverse effects (such as concentration problems, worsening memory and speech difficulties, among other possible factors experienced from multiple drug treatment) may have a negative effect on patients' cognition and communication levels.

All OOL domains showed a positive correlation with advancement in Hoehn and Yahr stage, corresponding to a lower QOL in all predictors of $\mathrm{QOL}$ in patients with more severe Parkinson's disease. The domains Mobility, ADL and Communication compounded a statistical significance both before and after intervention by the pharmacist. These findings confirm the findings of previous studies which showed that OOL was significantly better when the Hoehn and Yahr stage was lower. ${ }^{11-13}$

When assessing the results obtained from the relationship between the OOL domains and the Mental Test Score, a majority of negative correlations between the two variables were found. As the mean value for a particular $\mathrm{QOL}$ domain increased, a lower Mental Test Score was obtained. This signified that the lower the cognition level of the patient, the greater the problems encountered, corresponding to a poorer OOL. Mobility Before ( $\mathrm{p}=0.043), \mathrm{ADL}$ Before $(p=0.037)$, Cognition Before $(p=0.000)$, Cognition After $(p=0.000)$ and Communication Before $(p=0.031)$ were the domains that yielded a statistically significant negative correlation. These findings are in congruence with previous studies which concluded that better cognitive performance was independently associated with an overall better $\mathrm{QOL}$ in patients with Parkinson's disease. ${ }^{14}$ Conversely, the domains Stigma After, Social Support Before and Social Support After showed a positive correlation with the latter domain yielding a statistical significance, signifying that patients with a lower Mental Test Score scored less in this QOL domain. It was interesting to note that patients who experienced problems in cognition had better social support following pharmacist intervention (Social Support After). Prior to the pharmacist intervention (ie, Social Support Before), this relationship was not statistically significant.

When observing the set of results prior to the pharmacist's intervention, it was found that patients who were fully compliant with their medications had a generally better $\mathrm{QOL}$ than those who were not fully compliant with their medications. These patients scored the lowest mean values in five of the eight domains (ADL, Emotional, Cognition, Communication and Bodily Discomfort). Following the pharmacist's intervention, patients who never missed a dose had a better QOL than patients who rarely missed a dose for all domains except Cognition, signifying an improvement in the $\mathrm{QOL}$ of compliant patients. Despite the absence of a statistically significant relationship between $\mathrm{OOL}$ and compliance, a general trend was noticeable where patients who always took their medications as prescribed had a better health status. Improvement in $\mathrm{OOL}$ in compliant patients was furthermore noticeable at the second visit (ie, following the pharmacist's intervention) where patients showed a better health status than at the first visit. A possible explanation for this may be attributed to the pharmacist-run discussions with 
patients and their caregivers, as well as the use of the Treatment Medication Chart to aid in compliance and the patient leaflets with advice for ameliorating the $\mathrm{OOL}$ and the patient-caregiver relationship.

This study showed that pharmacist intervention had a positive impact on patients' OOL. The majority of patients were found to benefit from the Treatment Medication Chart since 32 patients found it 'always' or 'often' required, in addition to 30 patients and 27 patients who expressed this response for the QOL and Carers leaflets, respectively. The importance of pharmacist intervention in the clinical setting was further reinforced through the Intervention Evaluation Sheet (figure 6) where 30 patients deemed that the pharmacist's role was 'always' or 'often' required to affect treatment.

\section{DISCUSSION}

The fact that the incidence of being diagnosed with Parkinson's disease increases with advanced age, together with the patient's Hoehn and Yahr scale, implies a greater degree of disability. The decline in compliance with advanced age may be explained by the nature of Parkinson's disease itself, which is a chronic neurodegenerative disease with progressive impairment in mobility being accompanied by an increased risk of cognitive decline.

This study confirms the attestation that the inclusion of the pharmacist in the multidisciplinary team of healthcare professionals to affect treatment of patients with Parkinson's disease was indeed beneficial within the clinical setting in an outpatient setting. This was supported by the results obtained from the PDO-39 questionnaire which was found to be a suitable instrument for the assessment of patients' OOL status as it managed to highlight the significance of each $\mathrm{OOL}$ domain among different patients. For instance, the explanation for greater social support following the pharmacist's intervention in patients with problems in cognition may have been attributed to the pharmacist's explanation of the leaflet to caregivers and offering advice regarding the importance of their role in the patient's life, especially when some of these patients experienced cognition problems.

The study had limitations since time constraints limited pharmacist discussions with patients and caregivers and self-reporting was considered a subjective method for assessment of compliance and OOL status. Patients who lied about lack of compliance because

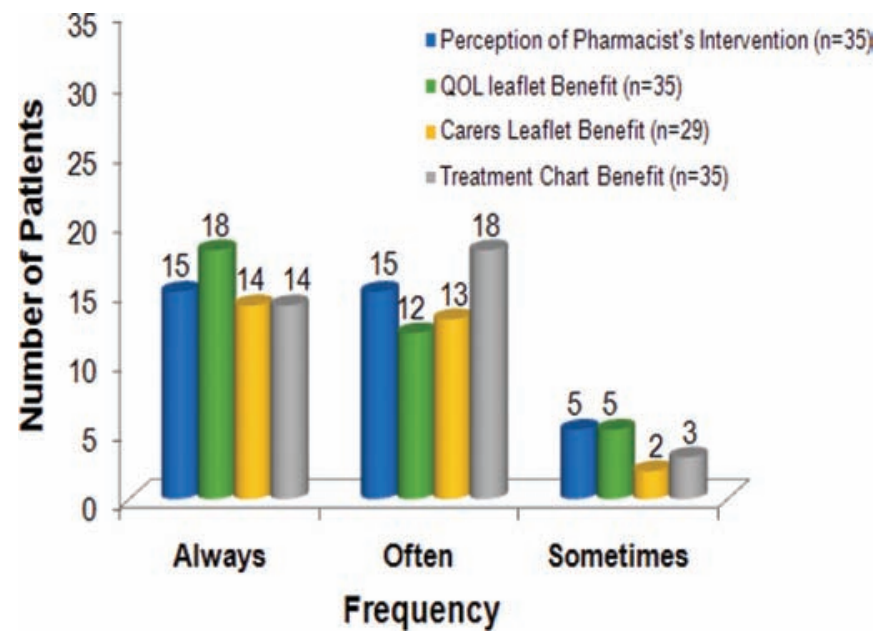

Figure 6 Patients' evaluation of role of pharmacist. they were embarrassed may have given misleading results. Such bias and error may also have arisen from the small sample size which may limit the generalisability of data.

It is recommended that this study should be extended to other clinics and hospitals in order to provide a larger sample of patients, yielding more statistically significant results. The study could be conducted over a longer time period with more follow-up visits, and more questions regarding the medications taken by patients should be introduced to attain more holistic results. Other proposals for further studies are the establishment of more support groups for patients and their caregivers and a greater focus on education through meetings, programmes using the media and updated websites in order to strengthen information about Parkinson's disease and help the general population be more informed about this disorder.

\section{CONCLUSIONS}

Each member of the multidisciplinary team has a fundamental role in the management of Parkinson's disease to help patients overcome the difficulties encountered. This study provides a promising prospect for the role of the pharmacist which is beneficial to both patients and caregivers. The inclusion of the pharmacist in the multidisciplinary team of healthcare professionals in the Clinic of Movement Disorders at Zammit Clapp Hospital may help to improve medication compliance and OOL for patients with Parkinson's disease and their caregivers.

Acknowledgements The author would like the thank Anthony Seracino Inglott, Lilian M Azzopardi and Janis Vella for their input into this article.

Competing interests None.

Patient consent Obtained.

Provenance and peer review Not commissioned; externally peer reviewed.

\section{References}

1. Zammit L, Azzopardi LM, Serracino-Inglott A, et al. Compliance and antihypertensive therapy. Clin Pharmacy Eur 2006;3:41-2.

2. Peto V, Jenkinson C, Fitzpatrick $\mathrm{R}$, et al. The development and validation of a short measure of functioning and well being for individuals with Parkinson's disease. Qual Life Res 1995;4:241-8.

3. Flores A. Assessment of patient compliance and patient perception of pain management II [dissertation]. Msida, Malta: University of Malta, 2005.

4. Pavon JM, Whitson HE, Okun MS. Parkinson's disease in women: a call for improved clinical studies and for comparative effectiveness research. Maturitas 2010;65:352-8.

5. Shulman LM, Gruber-Baldini AL, Anderson KE, et al. The evolution of disability in Parkinson's disease. Presented at the 57th annual meeting of the American Academy of Neurology, April 9-16, Miami Beach, Florida, 2005.

6. Carod-Artal FJ, Vargas AP, Martinez-Martin P. Determinants of quality of life in Brazilian patients with Parkinson's disease. Mov Disord 2007;22:1408-15.

7. Cubo E, Rojo A, Ramos S, et al. The importance of educational and psychological factors in Parkinson's disease quality of life. EurJ Neurol 2002;9:589-93.

8. Carod-Artal FJ, Ziomkowski S, Mourão Mesquita $\mathrm{H}$, et al. Anxiety and depression: main determinants of health-related quality of life in Brazilian patients with Parkinson's disease. Parkinsonism Relat Disord 2008;14:102-8.

9. Schrag A, Jahanshahi M, Quinn N. What contributes to quality of life in patients with Parkinson's disease? J Neurol Neurosurg Psychiatry 2000;69:308-12.

10. Moore O, Gurevich T, Korczyn AD, et al. Quality of sexual life in Parkinson's disease. Parkinsonism Relat Disord 2002;8:243-6.

11. Fitzsimmons B, Bunting LK. Parkinson's disease. Quality of life issues. Nurs Clin North Am 1993;28:807-18.

12. Clarke CE, Zobkiw RM, Gullaksen E. Quality of life and care in Parkinson's disease. Br J Clin Pract 1995;49:288-93.

13. Fukunaga $\mathrm{H}$, Kasai $\mathrm{T}$, Yoshidome $\mathrm{H}$. Clinical findings, status of care, comprehensive quality of life, daily life therapy and treatment at home in patients with Parkinson's disease. Eur Neurol 1997;38(Suppl 2):64-9.

14. Klepac N, Trkulja V, Relja M, et al. Is quality of life in non-demented Parkinson's disease patients related to cognitive performance? A clinic-based cross-sectional study. Eur J Neurol 2008;15:128-33. 